\title{
Anticoagulation in non-malignant portal vein thrombosis is safe and improves hepatic function
}

\author{
Bernhard Scheiner • Paul René Stammet · Sebastian Pokorny · Theresa Bucsics · Philipp Schwabl · \\ Andrea Brichta - Johannes Thaler · Katharina Lampichler - Ahmed Ba-Ssalamah · Cihan Ay · Arnulf Ferlitsch • \\ Michael Trauner · Mattias Mandorfer · Thomas Reiberger (D)
}

Received: 28 December 2017 / Accepted: 29 May 2018 / Published online: 18 June 2018

(C) The Author(s) 2018

\begin{abstract}
Summary
Background Non-malignant portal vein thrombosis (PVT) is common in patients with advanced liver disease. Anticoagulation (AC) increases the chances of recanalization and may improve liver function in patients with cirrhosis.

Aim We retrospectively assessed the course of nonmalignant PVT in patients receiving AC.

Methods Parameters related to hepatic injury (aspartate aminotransferase [AST]/alanine aminotrans-
\end{abstract}

Authors contributions Concept of the study (T. Reiberger; B. Scheiner; P. R. Stammet; S. Pokorny; J. Thaler; C. Ay; M. Mandorfer), extraction of data (P. R. Stammet; S. Pokorny; A. Brichta), writing of the manuscript (B. Scheiner; T. Reiberger; P. R. Stammet; S. Pokorny; M. Mandorfer), revision for important intellectual content (all authors), drafting of the manuscript (B. Scheiner; T. Reiberger; M. Mandorfer)

B. Scheiner · P. R. Stammet · S. Pokorny · T. Bucsics . P. Schwabl · A. Brichta · A. Ferlitsch · M. Trauner . M. Mandorfer $\cdot$ T. Reiberger $(\bowtie)$

Vienna Hepatic Hemodynamic Lab, Division of Gastroenterology and Hepatology, Department of Internal Medicine III, Medical University of Vienna, Waehringer Guertel 18-20, 1090 Vienna, Austria

thomas.reiberger@meduniwien.ac.at

B. Scheiner · P. R. Stammet · S. Pokorny · T. Bucsics . P. Schwabl · A. Ferlitsch · M. Trauner · M. Mandorfer • T. Reiberger

Vienna Hepatic Hemodynamic Laboratory, Medical University of Vienna, Vienna, Austria

J. Thaler · C. Ay

Clinical Division of Hematology and Hemostaseology,

Department of Medicine I, Medical University of Vienna, Vienna, Austria

K. Lampichler · A. Ba-Ssalamah

Department of Radiology, Medical University of Vienna,

Vienna, Austria ferase [ALT]), severity of disease (ascites) and synthesis function (albumin) as well as AC, rates of PVT regression/progression and $\mathrm{AC}$-associated complications were documented.

Results Among 122 patients with PVT, 51 patients with non-malignant PVT (27 incomplete, 24 complete) were included, 12 patients $(25 \%)$ received longterm AC therapy ( $\geq 9$ months) as compared to 36 patients without long-term AC. We observed a trend towards higher regression rates with long-term AC of $58 \%$ (vs. $28 \%$ without AC; $p=0.08$ ) and lower progression rates of $25 \%$ (vs. $42 \%$ without AC; $p=0.15$ ). In the subgroup of patients with decompensation prior to PVT diagnosis $(n=39)$, long-term AC $(n=10$, $25.6 \%)$ resulted in a significantly higher rate of PVT regression/resolution ( $70 \%$ vs. $24 \%, p=0.031)$. Interestingly, AST/ALT tended to decrease $(-19 \% /-16 \%)$ and the proportion of patients with ascites became lower (-33\%) with long-term AC (without AC: $\pm 0 \%$ ). Furthermore, there was a significant improvement in albumin levels $(+9 \% /+3.6 \mathrm{~g} / \mathrm{dl})$ when compared to patients without long-term AC $(-2 \% /-0.8 \mathrm{~g} / \mathrm{dl} ; p=0.04)$. Additionally, 10 patients were treated with direct oral anticoagulants (DOACs) for splanchnic vein thrombosis. Importantly, there were no AC-associated bleeding events in patients with conventional $\mathrm{AC}$ and one bleeding event in patients with DOAC treatment $(10 \%)$.

Conclusion Our findings support anticoagulation in patients with non-malignant PVT, since AC seems safe and associated with superior PVT regression rates and might also decrease hepatic injury and improve liver synthesis.

Keywords Liver diseases · Portal vein · Venous thrombosis · Anticoagulants 


\section{Study highlights}

- Long-term anticoagulation (AC) in patients with portal vein thrombosis is recommended by international guidelines to improve recanalization rates.

- In this study, long-term AC was associated with a trend towards higher portal vein thrombosis (PVT) regression rates, which attained statistical significance in patients with decompensated cirrhosis.

- Furthermore, long-term AC was associated with significant improvements in hepatic synthesis function (albumin).

- In patients with liver cirrhosis AC appears to be safe, as no AC-associated bleeding events occurred in patients with conventional $\mathrm{AC}$ and only one bleeding event in DOAC patients.

\section{Introduction}

Cirrhosis can develop from virtually all forms of chronic liver disease with alcoholic liver disease and chronic viral hepatitis being the most common etiologies in developed countries [1]. Patients with cirrhosis are at increased risk for gastrointestinal bleeding, mainly from esophageal varices or portal hypertensive gastropathy $[2,3]$. In contrast to the dogma of auto-anticoagulation in cirrhosis, venous thrombosis is more common in patients with cirrhosis than in the general population $[4,5]$. There is a 7.3 -fold increased risk of developing portal vein thrombosis (PVT) with cirrhosis. This represents a severe complication in patients with liver cirrhosis that may lead to intestinal infarction and preclude the option for orthotopic liver transplantation [6, 7]. The prevalence of PVT is up to $23.3 \%$ in liver transplantation candidates without hepatocellular carcinoma (HCC) and the yearly incidence of non-malignant PVT is estimated to be between $7-11 \%$ in patients with cirrhosis.

Although partial PVT in patients with cirrhosis may resolve spontaneously $[8,9]$, the majority of patients (48-70\%) show progression of PVT within 2 years [8, 10]. Thus, anticoagulation with low molecular weight heparin (LMWH) or vitamin $\mathrm{K}$ antagonists (VKA) has been used for treatment of cirrhotic PVT with reported recanalization rates of 55-75\% [10-14] when given for about 6 months. Since PVT might recur in up to $38 \%$ of cirrhotic patients when anticoagulation is stopped early after PVT resolution [9, 13], long-term anticoagulation has been suggested by recent European Association for the Study of the Liver (EASL) guidelines [15], particularly in liver transplantation candidates.

Since PVT can cause portal hypertension-related bleeding and anticoagulation may further increase the risk of bleeding events, the safety of anticoagulation therapy needs to be specifically addressed in patients with cirrhosis; however, in previous studies the incidence and severity of bleeding events related to anticoagulation with LMWH or VKA was low [10-14].
Next to the efficacy of anticoagulation to prevent and resolve PVT, there are experimental data supporting a beneficial impact on liver synthesis function and fibrosis [16]. In a prospective clinical trial, the administration of prophylactic LMWH did not only prevent PVT in liver transplantation candidates but also decreased hepatic decompensation and improved survival [17].

Thus, this study aimed to assess (i) regression rates of non-malignant PVT with and without anticoagulation and (ii) the evolution of hepatic inflammation and liver function in patients with PVT. Furthermore (iii) we provide a summary of the existing literature on this topic.

\section{Patients and methods}

\section{Study design and inclusion of subjects}

This study was a retrospective analysis on the efficacy of anticoagulation for regression of PVT and its impact on hepatic inflammation and liver function. The specific inclusion criteria for this study were patients aged $\geq 18$ years with a diagnosis of cirrhosis and concomitant PVT. We excluded patients with malignant PVT and patients without sufficient clinical information at the time of PVT diagnosis and on the clinical course thereafter. Furthermore, data on direct oral anticoagulants (DOACs) used in a small cohort of patients for treatment of splanchnic vein thrombosis are reported.

\section{Clinical and radiological parameters}

Clinical data was collected from patients' medical records. Radiological images by computed tomography (CT), magnetic resonance imaging (MRI) and sonography were reviewed by a second expert radiologist in case PVT diagnosis or extension of thrombus was unclear. Demographic characteristics and comorbidities were recorded at PVT diagnosis. In addition, we longitudinally assessed the use of anticoagulation therapy with LMWH or phenprocoumon. The course of PVT was recorded as (i) regression, (ii) stable or (iii) progression. Ascites and hepatic encephalopathy were graded according to the Child-Pugh score at PVT diagnosis and at follow-up. Moreover, bleeding events during follow-up were recorded.

\section{Laboratory parameters}

Serum levels of creatinine, bilirubin, albumin, and liver enzymes were recorded at PVT diagnosis and at follow-up. Furthermore, prothrombin time and International Normalized Ratio (INR) were assessed at both time points (baseline and follow-up) and Model for End-stage Liver Disease (MELD) and Child-Pugh scores were calculated. 


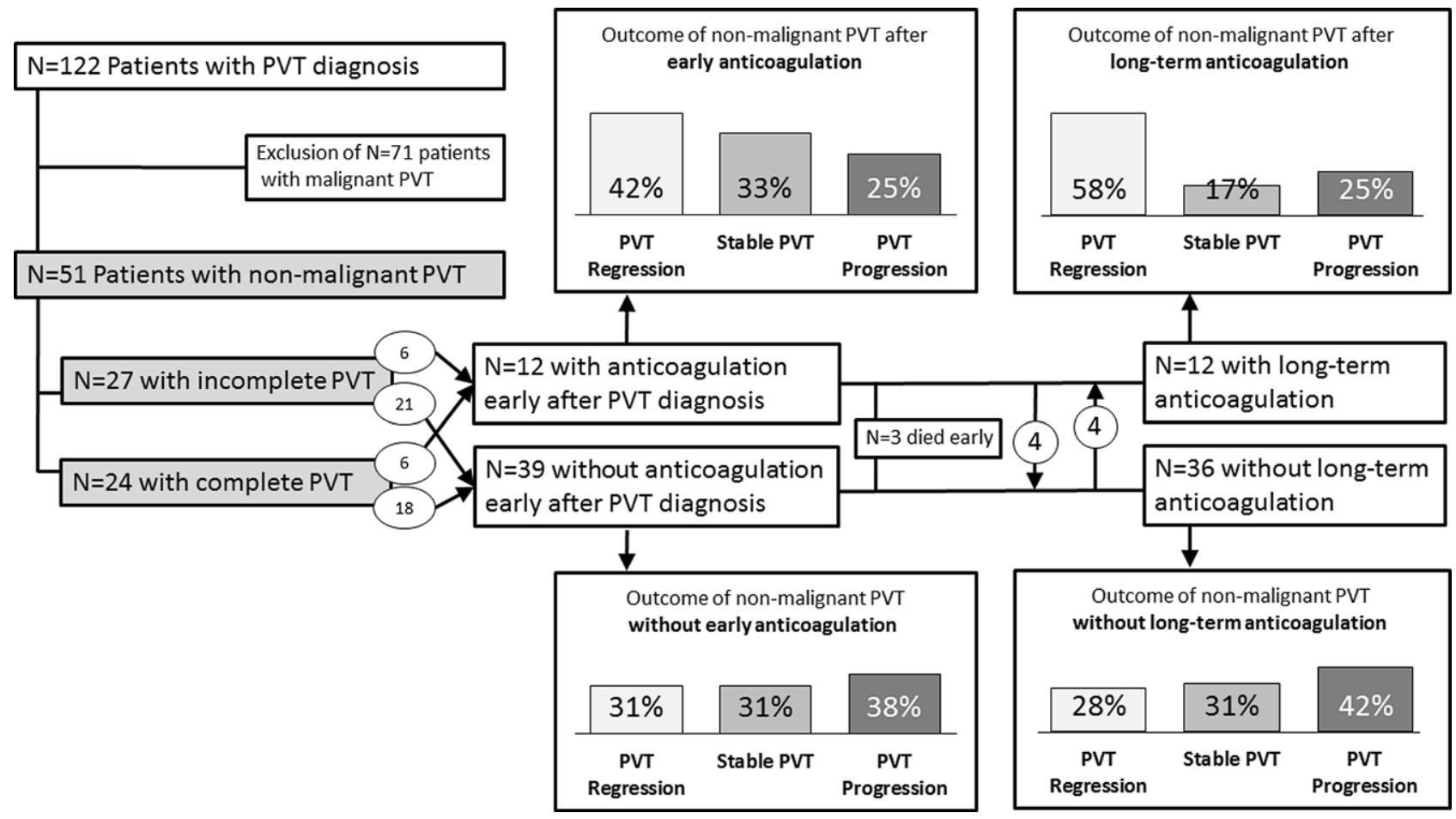

Fig. 1 Consort flowchart and rates of PVT resolution, stabilization and progression

\section{Definitions, time points and groups}

Patient data were assessed at baseline and at last follow-up (as defined by the last available imaging). For analysis, patients were devided into groups two times: (1) patients receiving early anticoagulation vs. patients not receiving anticoagulation/delayed start of anticoagulation and (2) patients receiving longterm anticoagulation vs. no anticoagulation/shortterm anticoagulation. Long-term anticoagulation was defined as at least 9 months and until the end of follow-up. Major bleeding was defined according to the recent Baveno V consensus workshop [18]: active bleeding at endoscopy after the start of a specific drug treatment, with a drop of $\geq 3 \mathrm{~g} / \mathrm{dl}$ of hemoglobin or bleeding requiring blood transfusion with an adjusted blood transfusion requirement index (ABRI) $\geq 0.75$ at any time point.

\section{Statistics}

All statistical analyses were performed using IBM SPSS Statistics 23 (SPSS, Armonk, NY, USA) and/or GraphPad Prism (version 6.00, GraphPad Software, La Jolla, CA, USA). Continuous variables were reported as mean \pm standard deviation or median (interquartile range). Categorical variables were reported as number (proportion) of patients with/without the certain characteristic. The clinical course of PVT was described by calculating the proportion of patients that showed (i) regression, (ii) stable PVT and (iii) progression of PVT. We used the parametric Student's t-test for group comparisons if applicable. Otherwise, nonparametric tests were used (e.g. Wilcoxon MannWhitney U-test). Group comparisons of categorical variables were performed using either Pearson's $\chi^{2}$-test or Fisher's exact test. $P$-values $<0.05$ were considered statistically significant.

\section{Results}

\section{Patients' characteristics}

We identified a total of 122 patients diagnosed with PVT at our center, whereby 71 patients had to be excluded due to underlying malignancies. Thus, 51 patients with non-malignant PVT were included in this analysis (Fig. 1). The majority of patients were male $(62.7 \%)$ with a mean age of $52.9 \pm 12.5$ years. The main etiology of liver disease was alcohol abuse in 24 patients $(47.1 \%)$. Non-selective beta-blockers (NSBB) were used by 22 patients $(43.1 \%)$ and most were on proton pump inhibitor (PPI) therapy ( $n=45$, 88.2\%). Patients were followed for a median time of 44.1 (14.0-79.1) months. Anticoagulation treatment ( $n=16$ patients) was maintained for a median time of 12.0 (8.7-29.0) months (Table 1).

\section{Clinical presentation at PVT diagnosis}

At PVT diagnosis, 14 patients (27.5\%) had compensated cirrhosis (Child-Pugh stage A), while 37 patients had decompensated cirrhosis (Child-Pugh stage B: $n=19,37.3 \%$; Child-Pugh stage C: $n=18,35.3 \%$ ). The 
Table 1 Patient characteristics of patients with non-malignant PVT ( $(\%)$; mean \pm SD; median (IQR))

\begin{tabular}{|c|c|c|c|}
\hline \multicolumn{4}{|l|}{ Patients: $n=51(100 \%)$} \\
\hline Age (years) & $52.9 \pm 12.5$ & $N S B B$ & $22(43.1 \%)$ \\
\hline Gender (M/F, \%M) & $32 / 19(62.7 \%)$ & PPI & $45(88.2 \%)$ \\
\hline \multicolumn{2}{|l|}{ Etiology of liver disease } & Ascites & $34(66.7 \%)$ \\
\hline Alcohol & $24(47.1 \%)$ & Overt HE & $7(13.7 \%)$ \\
\hline Viral & $6(11.8 \%)$ & Bilirubin (mg/dl) & $1.78(1.88)$ \\
\hline Other & $21(41.1 \%)$ & Albumin (g/dl) & $33.3 \pm 7.5$ \\
\hline Child-Pugh A & $14(27.5 \%)$ & INR & $1.45 \pm 0.32$ \\
\hline Child-Pugh B & $19(37.3 \%)$ & $C R P(m g / d l)$ & $1.26(2.99)$ \\
\hline Child-Pugh C & $18(35.2 \%)$ & Platelets (G/I) & $113(175)$ \\
\hline Child-Pugh score & $8.6 \pm 2.7$ & \multicolumn{2}{|l|}{ Severity of PVT } \\
\hline MELD & $13.6 \pm 6.5$ & Partial PVT & $27(52.9 \%)$ \\
\hline Diabetes & $5(9.8 \%)$ & Complete PVT & $24(47.1 \%)$ \\
\hline Arterial hypertension & $6(11.8 \%)$ & Follow-up (months) & $44.1(14.0-79.1)$ \\
\hline Varices & $39(76.5 \%)$ & \multirow{2}{*}{$\begin{array}{l}\text { Duration of anticoagulation (months) in } \\
n=16 \text { patients }\end{array}$} & \multirow[t]{2}{*}{$12.0(8.7-29.0)$} \\
\hline Previous variceal bleeding & $21(41.2 \%)$ & & \\
\hline
\end{tabular}

mean MELD score was $13.6 \pm 6.5$ points. Most patients had esophageal varices $(n=39,76.5 \%)$ and 21 patients (41.2\%) presented with a history of variceal bleeding. Two thirds of patients $(n=34,66.7 \%)$ showed ascites at the time of PVT diagnosis, while overt hepatic encephalopathy was less common $(n=7,13.7 \%)$. Half of patients ( $n=27,52.9 \%)$ showed incomplete PVT and $n=24$ patients $(47.1 \%)$ had complete PVT (Table 1 ).

\section{Early anticoagulation and regression rates of PVT}

Following endoscopic evaluation, $n=12$ patients $(23.5 \%)$ were started with early $(<10$ days after PVT diagnosis) anticoagulation (Fig. 1). This decision to start early anticoagulation was at the discretion of the treating physician. In the group of patients being started with anticoagulation the proportion of female patients was higher $(p=0.04)$ and more patients without anticoagulation were taking NSBB $(p=0.03)$. The other baseline characteristics of patients receiving vs. not receiving early anticoagulation did not differ. Anticoagulation was initially achieved with LMWH and followed by oral anticoagulation with phenprocoumon (target INR levels 1.5-2.0).

When comparing PVT regression rates of patients with $(n=12)$ and without $(n=39)$ early AC, we found higher PVT regression rates $(42 \%$ vs. $31 \%)$ and a lower rate of PVT progression (25\% vs. $38 \%$ ); however this result did not attain statistical significance $(p=0.67)$.

\section{Long-term anticoagulation and outcome of PVT}

While three patients (5.9\%) died early during followup and were excluded from further analysis, in four patients $(8.3 \%)$ anticoagulation was stopped and in four other patients anticoagulation was started during follow-up. Finally, 12 patients $(25.0 \%)$ were kept on long-term anticoagulation therapy (AC, Fig. 1). Patients receiving long-term AC showed a trend towards higher PVT regression rates $(58 \%$ vs. $28 \%$ in patients receiving vs. not receiving long-term $\mathrm{AC} ; p=0.08$ ).

\section{Long-term anticoagulation and outcome of PVT in patients with decompensated cirrhosis}

In total, 39 patients experienced decompensation of liver disease (history of or current ascites, hepatic encephalopathy or variceal bleeding) prior to PVT diagnosis. In this subgroup, a quarter of patients $(n=10$, $25.6 \%)$ received long-term anticoagulation which resulted in a significantly higher rate of PVT regression when compared to patients without long-term anticoagulation ( $70 \%$ vs. $24 \%, p=0.031$; Table 2 ).

\section{Effects of anticoagulation on serum transaminases}

At PVT diagnosis, median levels of AST (35 [IQR:33] vs. 34 [IQR:36] IU/ml; $p=0.74$ ) and ALT (34 [IQR:25] vs. 24 [IQR:23] IU $/ \mathrm{ml} ; p=0.33$ ) were similar in patients who subsequently received and in those who did not receive long-term $\mathrm{AC}$ (Fig. 2a, b). During long-term AC, liver enzymes decreased to median values of AST: 32 (IQR:24) IU/ml (-19\%) and ALT: 26 (IQR:19) IU/ml (-16\%) but increased without AC to AST: 47 (IQR:41) IU/ml (+17\%) and ALT: 29 (IQR:25) IU/ml (+3\%). However, the differences in the change of AST/ALT between patients with versus without long-term AC did not attain significance (AST: $p=0.43$; ALT: $p=0.15$ ).

\section{Impact of anticoagulation on ascites control and liver synthetic function}

Interestingly, in four out of nine patients who had ascites at time of PVT diagnosis, ascites control was 
Table 2 Development of portal vein thrombosis in patients with decompensated cirrhosis with vs. without long-term anticoagulation $(n(\%))$

\begin{tabular}{|l|l|l|l|}
\hline & No long-term anticoagulation $(n=29)$ & Long-term anticoagulation $(n=10)$ & $p$-value \\
\hline Regression/resolution of PVT & $7(24.1)$ & $7(70.0)$ & $p=0.031$ \\
\hline Stabilization of PVT & $11(37.9)$ & $1(10.0)$ & \\
\hline Progression of PVT & $11(37.9)$ & $2(20.0)$ & \\
\hline
\end{tabular}

Table 3 Bleeding events and sites during follow-up ( $n(\%))$

\begin{tabular}{|l|l|l|l|}
\hline Bleeding episodes & Anticoagulation $(n=16)$ & No anticoagulation $(n=35)$ & $p$-value \\
\hline Patients experiencing bleeding & 0 & 16 & - \\
\hline Bleeding sites & $0(-)$ & $8(22.9 \%)$ & 0.045 \\
\hline Gastric varices & & & - \\
\hline Esophageal varices & $3(19 \%)$ & $6(38 \%)$ \\
\hline Gastric angiodysplasia & & $1(6 \%)$ \\
\hline Esophageal ulcus post EBL & $2(13 \%)$ \\
\hline Portal hypertensive gastropathy & $2(12 \%)$ \\
\hline Duodenal ulcus & $2(12 \%)$ \\
\hline
\end{tabular}

Table 4 Patient characteristics and outcomes of patients with splanchnic vein thrombosis treated with direct oral anticoagulants (DOACs; $n(\%)$; mean \pm SD; median (IQR))

\begin{tabular}{|c|c|c|c|}
\hline \multicolumn{4}{|l|}{ Patients: $n=10(100 \%)$} \\
\hline Age (years) & $50 \pm 18$ & Previous variceal bleeding & $2(20 \%)$ \\
\hline Gender $(M / F, \% M)$ & $2 / 8(20 \%)$ & Bilirubin (mg/dl) & $0.49(0.31)$ \\
\hline Liver cirrhosis & $3(30 \%)$ & Albumin (g/dl) & $39.1 \pm 5.0$ \\
\hline Occlusion (partial/complete, \% complete) & $4 / 6(60 \%)$ & INR & $1.21 \pm 0.30$ \\
\hline \multicolumn{2}{|l|}{ Extension } & \multicolumn{2}{|l|}{ DOAC used } \\
\hline V. portae & $4(40 \%)$ & Edoxaban (30 or $60 \mathrm{mg}$ once daily) & $4(40 \%)$ \\
\hline V. portae + V. mes. sup & $2(20 \%)$ & Apixaban (5 mg twice daily) & $3(30 \%)$ \\
\hline V. portae + V. mes. sup. + V. lienalis & $3(30 \%)$ & Rivaroxaban (10 mg once daily) & $2(20 \%)$ \\
\hline V. lienalis & $1(10 \%)$ & Dabigatran (110 mg twice daily) & $1(10 \%)$ \\
\hline Varices at treatment start & $8(80 \%)$ & Follow-up on DOAC treatment (months) & $9.2(5.4-13.7)$ \\
\hline \multicolumn{4}{|l|}{ Outcome } \\
\hline \multicolumn{3}{|l|}{ Regression/resolution of PVT } & $2(20 \%)$ \\
\hline \multicolumn{3}{|c|}{ Stable PVT including patients with unchanged cavernous transformation of portal vein } & $8(80 \%)$ \\
\hline \multicolumn{3}{|l|}{ Bleeding events during DOAC treatment } & $1(10 \%)$ \\
\hline
\end{tabular}

achieved after long-term AC. In contrast, in the group of patients without long-term AC, the number of patients with ascites remained unchanged $(p=0.24$; Fig. 2c).

When assessing album levels as a marker of liver synthesis, there was no difference in albumin levels between patients with and without subsequent AC: 31.1 (IQR:13.6) vs. 35.1 (IQR:8.7) g/dl $(p=0.79)$ at the time of PVT diagnosis. However, after longterm AC, serum albumin levels increased to 43.8 (IQR:14.5) g/dl, while albumin levels remained unchanged in patients without AC during follow-up: 32.2 (IQR:10.5) g/dl. Importantly, albumin levels significantly improved in patients receiving long-term AC when compared to patients without long-term AC $(+9 \%$ or $+3.6 \mathrm{~g} / \mathrm{dl}$ vs. $-2 \%$ or $-0.8 \mathrm{~g} / \mathrm{dl} ; p=0.04$; Fig. $2 \mathrm{~d})$.

\section{Safety of anticoagulation in patients with non-} malignant PVT

At baseline, 20 patients reported a history of variceal bleeding, 4 patients (33.3\%) in the long-term AC group and 16 patients $(44.4 \%)$ in the without long-term AC group $(p=0.50)$. In total, 16 bleeding episodes were registered in 8 patients $(15.7 \%)$ within the study period and 2 patients $(2.4 \%)$ died because of variceal bleeding (Table 3). Importantly, no bleeding episodes occurred in patients receiving $\mathrm{AC}(p=0.045)$.

\section{DOAC treatment for splanchnic vein thrombosis}

Additionally, 10 patients treated with DOACs for splanchnic vein thrombosis are reported separately (Table 4 ). The majority of patients were female (80\%) 


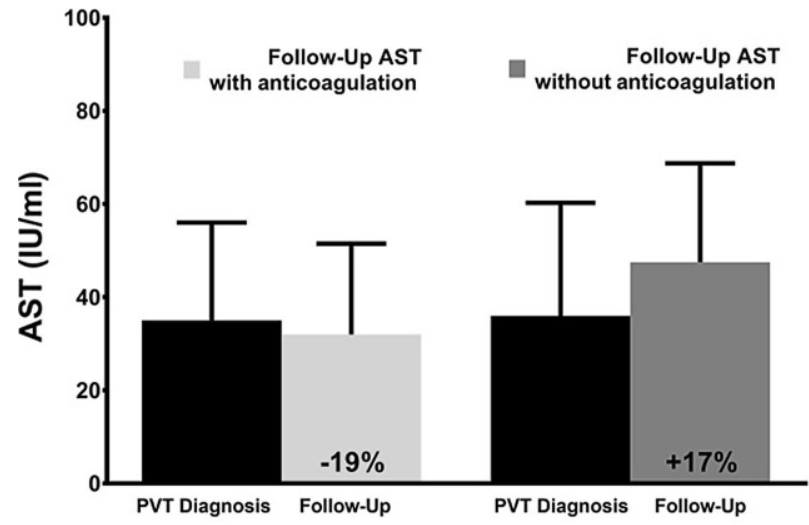

a

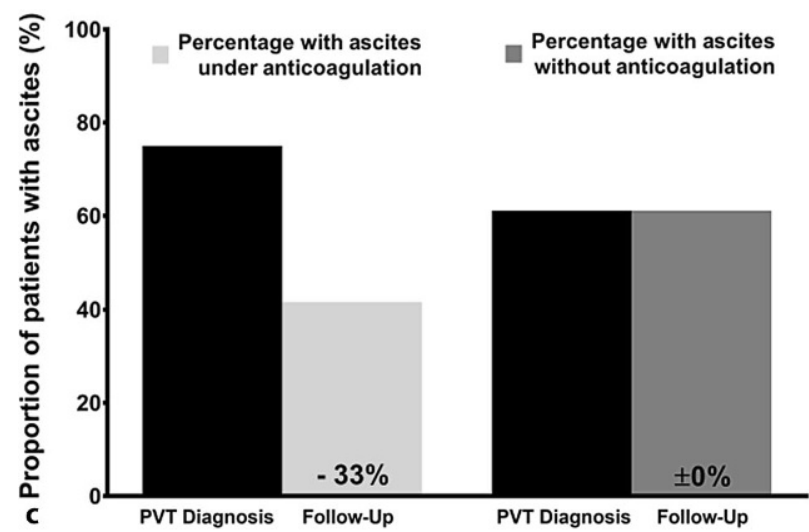

Fig. 2 Course of nonmalignant PVT in patients with and without long-term anticoagulation. (a, b) Levels of serum aminotransferases at PVT diagnosis and during follow-up are shown separately for the groups of patients with long-term anticoagulation versus without long-term anticoagulation. While both aspartate aminotransferase (AST) and alanine aminotransferase (ALT) tended to decrease with long-term anticoagulation $(-19 \%,-16 \%)$, AST/ALT increased in patients without an-

and had a non-cirrhotic splanchnic vein thrombosis $(70 \%)$. More detailed baseline characteristics can be found in Table 4 . While 4 patients $(40 \%)$ were treated with edoxaban (3 patients: $30 \mathrm{mg}$ once daily; 1 patient: $60 \mathrm{mg}$ once daily), 3 patients $(30 \%)$ received apixaban (5 mg twice daily), 2 patients (20\%) rivaroxaban (10 mg once daily) and 1 patient (10\%) dabigatran (110 mg twice daily). The majority of patients were switched to DOACs in the setting of chronic PVT with cavernous transformation in order to prevent progression/extension of splanchnic vein thrombosis and potentially to improve splanchnic, intestinal and hepatic microcirculation. In this setting AC therapy can of course not recanalize PVT and treatment response can only be measured as non-progression of PVT. Therefore, comparison of treatment success between DOAC patients and patients treated with conventional AC was not performed. Median follow-up on DOAC treatment was 9.2 (IQR: 5.4-13-7) months. One bleeding event (i.e. bleeding from por-

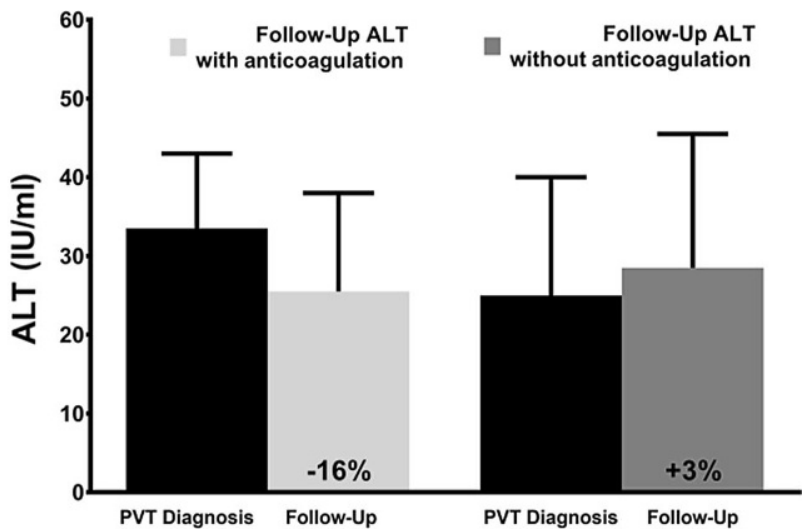

b

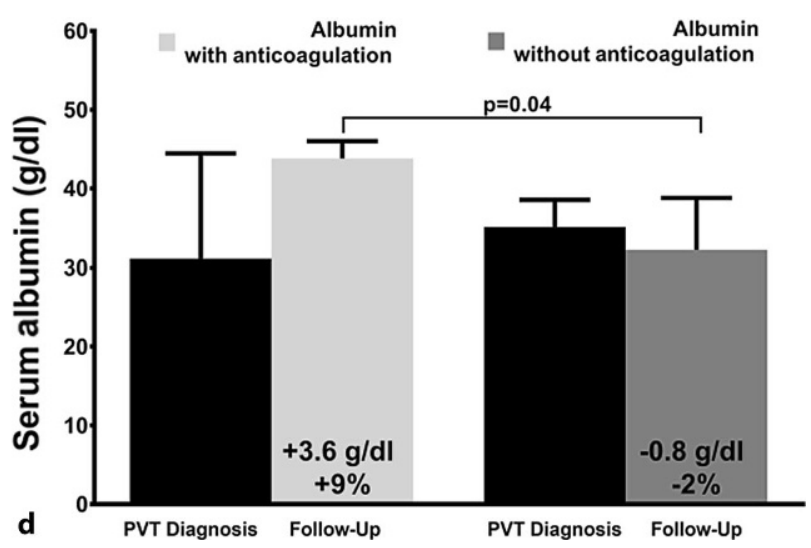

ticoagulation $(+17 \%,+3 \%)$. c While the proportion of patients with ascites decreased with long-term anticoagulation (from 9 to 5 patients, $-33 \%$ ), the number of patients with ascites remained unchanged without anticoagulation. d Serum albumin levels increased with long-term anticoagulation $(+3.6 \mathrm{~g} / \mathrm{dl}$, $+9 \%)$ but remained stable in patients without anticoagulation $(-0.8 \mathrm{~g} / \mathrm{dl},-2 \%)$

tal hypertensive gastropathy) occurred during DOAC treatment.

\section{Review of the literature on the course of PVT and efficacy of anticoagulation}

Of the studies eight (five prospective cohort studies, one prospective randomized cohort study and two retrospective cohort studies) were identified that reported the outcome of PVT and data on the efficacy and safety of anticoagulation therapy in cirrhotic patients with non-malignant PVT [8-14, 19] (Table 5). Most studies used either LMWH (mostly nadroparin or enoxaparin) or VKA (mostly after starting AC with LMWH first). The reported PVT resolution rates ranged from $42.1 \%$ to $82 \%$ and were consistantly higher with anticoagulation [11, 14]. The incidence of bleeding was low and the severity of the bleeding events reported was mild or moderate [10, 12, 14, 19]. A VKA therapy was usually adapted to reach an INR between 2-3 [11, 14]. 


\begin{tabular}{|c|c|c|c|c|c|c|c|c|}
\hline 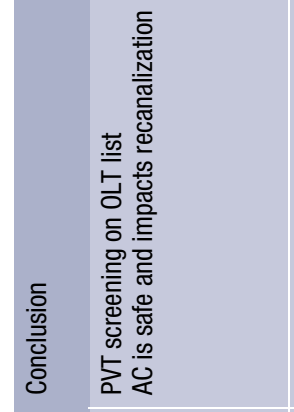 & 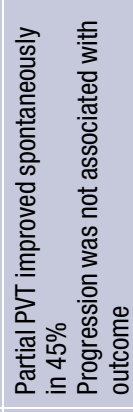 & 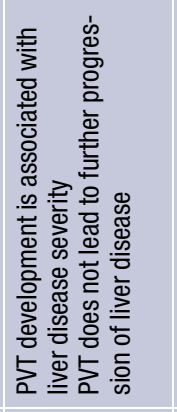 & 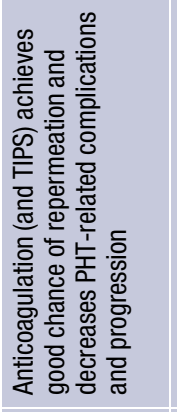 & 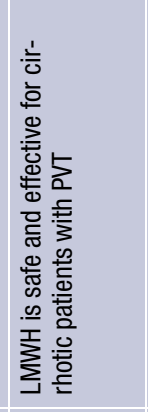 & 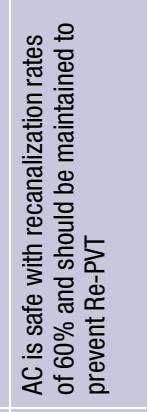 & 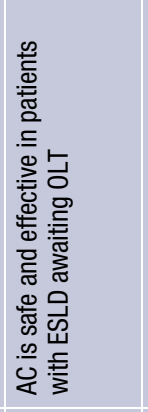 & 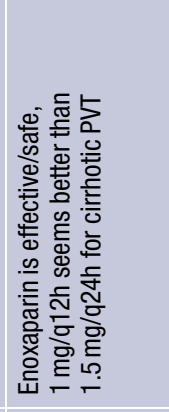 & 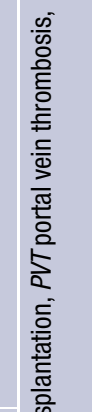 \\
\hline 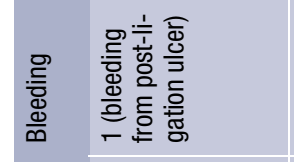 & 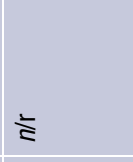 & 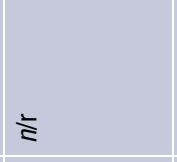 & 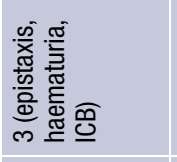 & 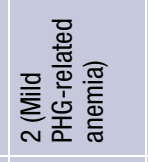 & 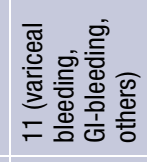 & 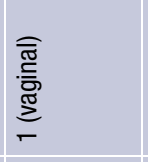 & 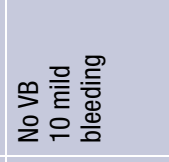 & 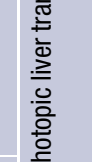 \\
\hline 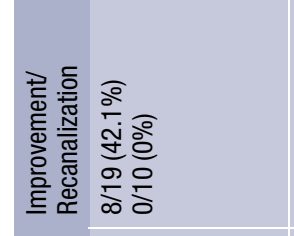 & 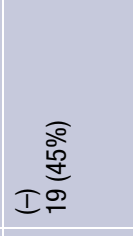 & 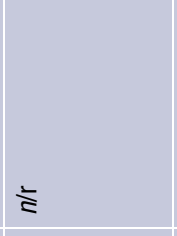 & 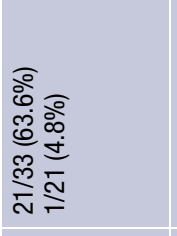 & 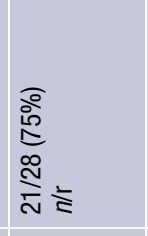 & 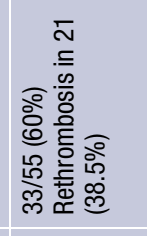 & $\begin{array}{l}\bar{o} \\
\text { ò } \\
\infty \\
\stackrel{\infty}{N} \\
\text { N }\end{array}$ & 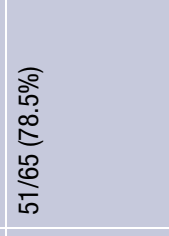 & 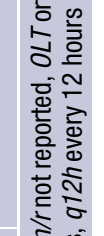 \\
\hline$\stackrel{\Xi}{\underline{\underline{I}}}$ & $\underset{\sim}{E}$ & $\underset{\mathcal{F}}{E}$ & $\begin{array}{l}E \\
\stackrel{E}{N}\end{array}$ & $\stackrel{E}{E}$ & Е & $\frac{E}{0}$ & E & 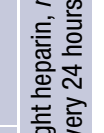 \\
\hline 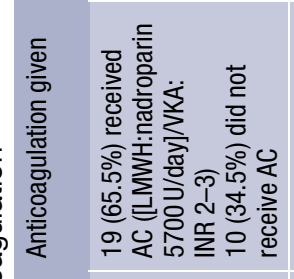 & 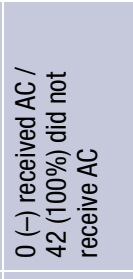 & 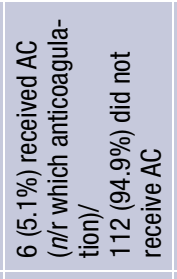 & 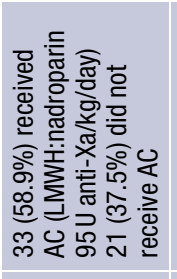 & 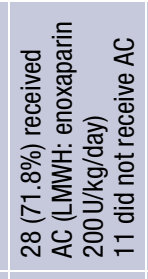 & 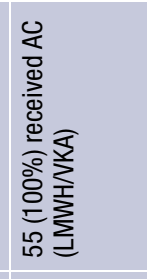 & 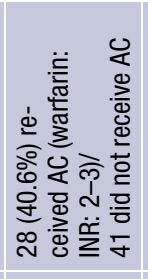 & 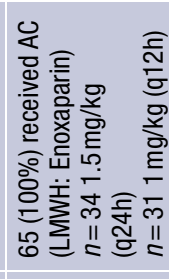 & 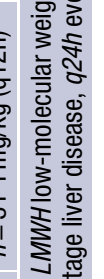 \\
\hline 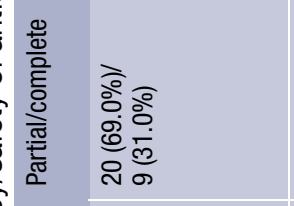 & 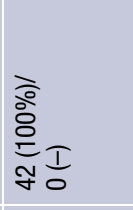 & 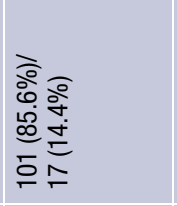 & 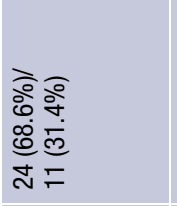 & 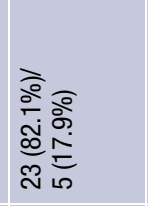 & 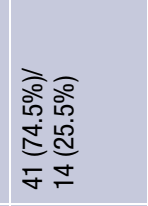 & $\lesssim$ & 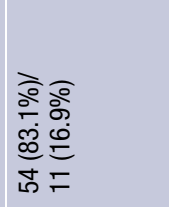 & 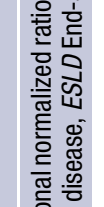 \\
\hline$\frac{0}{2}$ & 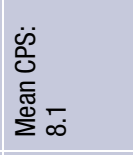 & 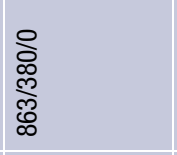 & 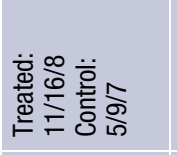 & $\begin{array}{l}\stackrel{\circ}{\circ} \\
\dot{0} \\
\dot{0} \\
\dot{\infty}\end{array}$ & 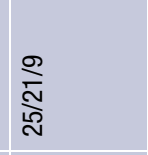 & 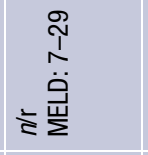 & 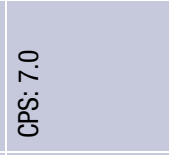 & 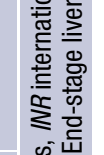 \\
\hline 点 & ָี & $\begin{array}{l}\stackrel{m}{\sim} \\
\stackrel{\infty}{\infty} \\
\stackrel{+}{F}\end{array}$ & 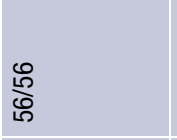 & 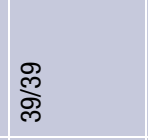 & 㟔 & $\begin{array}{l}\hat{\tilde{m}} \\
\stackrel{\leftrightarrow}{8}\end{array}$ & 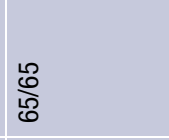 & 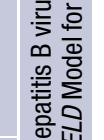 \\
\hline 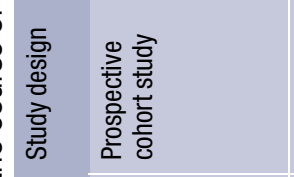 & 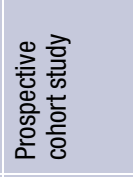 & 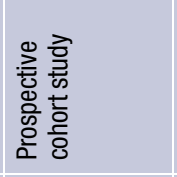 & 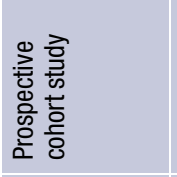 & 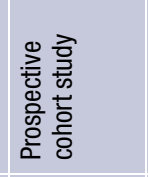 & 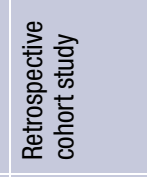 & 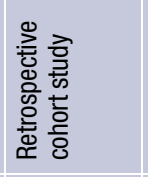 & 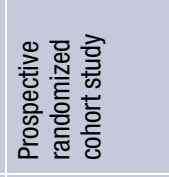 & 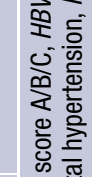 \\
\hline 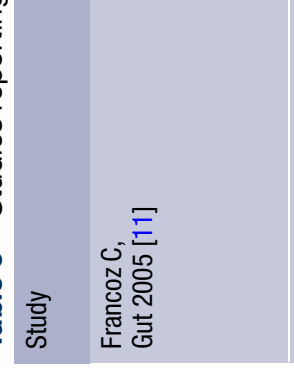 & 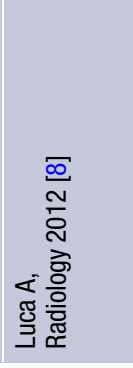 & 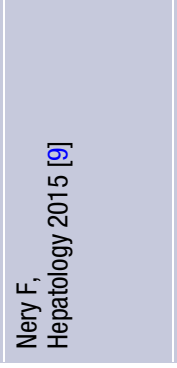 & 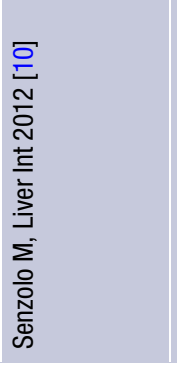 & 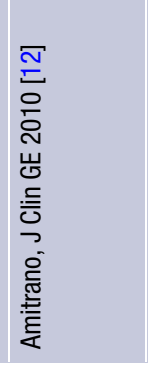 & 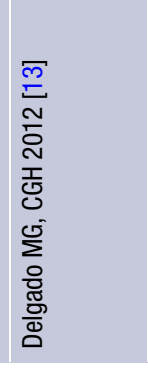 & 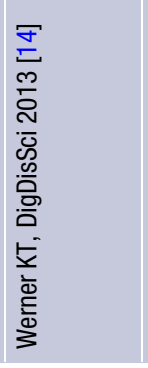 & 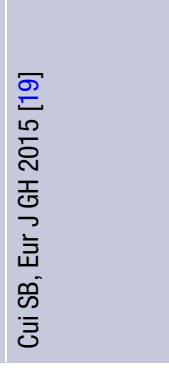 & 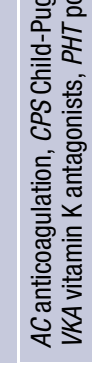 \\
\hline
\end{tabular}




\section{Discussion}

In our series of patients with non-malignant PVT, anticoagulation resulted in a trend towards higher regression rates and a decreased risk of PVT progression. While the risk of PVT progression could not be completely abolished by the use of anticoagulation, we observed beneficial impacts on hepatic inflammation and liver function: Interestingly, in patients with long-term anticoagulation, the levels of transaminases (AST/ALT) decreased numerically and albumin as a parameter of liver synthesis function increased significantly. Furthermore, in a subgroup analysis including patients with previously decompensated liver disease, we observed statistically significant higher PVT regression rates with long-term anticoagulation. This is especially important as patients with decompensated cirrhosis are at increased risk of developing complications and there is a pronounced need for successful treatment of PVT [20-22]. Importantly, the incidence of gastrointestinal bleeding was not increased with anticoagulation and even in patients presenting with previous variceal bleeding at PVT diagnosis no re-bleeding occurred during follow-up. It can be concluded that $\mathrm{AC}$ in cirrhotic patients even with previous variceal bleeding can be started safely after endoscopic exclusion of potential highrisk bleeding sites. In our clinic, if high-risk varices are present at screening, anticoagulation therapy is started after appropriate treatment of varices (verified hemodynamic response to NSBB therapy or endoscopic band ligation).

The observed PVT regression rate in our patient series was $58 \%$ when anticoagulation was given as long-term treatment. This finding is consistent with previous studies reporting regression/resolution rates ranging from $42.1 \%$ [11] up to $82 \%$ [14]. While the proportion of patients achieving regression of PVT on anticoagulation was numerically higher in the whole cohort and statistically significantly higher in patients with decompensated liver disease, the low number of patients with compensated liver disease treated with anticoagulation $(n=2)$ limited the statistical power of our analysis in the whole cohort, and thus, the difference in resolution/recanalization rate did not attain statistical significance. Nevertheless, the numerically higher PVT regression/resolution rates with anticoagulation represent a clinically relevant effect size and anticoagulation should be offered to all patients with PVT in the absence of absolute contraindications (such as active gastrointestinal bleeding or a history of intracranial bleeding).

While bleeding rates and severe bleeding events were not consistently reported across all studies, bleedings were usually mild and variceal bleeding was rare [10, 11, 13, 14]. In our study, no bleeding occurred in patients receiving conventional AC. In patients treated with DOACs one bleeding episode occurred; however, bleeding was controlled endo- scopically (with argon plasma coagulation) and treatment could be restarted. This is in line with the results of another study, in which the authors did not observe an increased bleeding risk in patients with cirrhosis receiving novel direct oral anticoagulants (rivaroxaban or apixaban), when compared to LMWH/ phenprocoumon [23].

The safety of anticoagulation in patients with PVT could also be explained by potential beneficial effects on intrahepatic vascular resistance, which were recently described in an experimental study in cirrhotic rats treated with enoxaparin [16]. Interestingly, longterm administration of enoxaparin reduced portal pressure by inhibiting hepatic stellate cell activation (dynamic component of portal hypertension), decreasing liver fibrosis (structural component of portal hypertension) and by prevention of intrasinusoidal microthrombosis [16].

We found a decrease in serum transaminases with long-term anticoagulation, as both AST and ALT levels dropped (by $-19 \%$ and $-16 \%$, respectively) while AST/ALT increased without long-term anticoagulation $(+17 \% /+3 \%)$. This suggests that the beneficial effects of enoxaparin described in the experimental study by Cerini et al. [16] might also lead to a decrease in hepatic necroinflammation in cirrhotic patients with PVT. Most interestingly, long-term anticoagulation was also associated with a better control of ascites during follow-up, as four out of nine patients with ascites at PVT diagnosis achieved control of ascites, while the number of patients with ascites remained stable in patients without long-term AC. In addition, we also found a significant increase in serum albumin under long-term anticoagulation $(+3.6 \mathrm{~g} / \mathrm{dl} /+9 \%)$ while serum albumin levels were almost unchanged in patients without long-term anticoagulation.

Our findings indicate that anticoagulation therapy leads to improvements in liver dysfunction and is in line with a previous study showing a decrease in hepatic decompensation with LMWH [17]. In line with our subgroup analysis, in the latter study, all patients were already decompensated at start of LMWH (Child-Pugh B7-C10 was an inclusion criterion) and no patients had PVT at inclusion. The study was designed to assess if prophylactic LMWH can prevent PVT, which was the case, but the even more interesting findings were the delay in hepatic decompensation during enoxaparin treatment $(11.7 \%$ vs. $59.6 \%$ in the control group) and increased survival rate in the enoxaparin treated group [17].

While the study by Villa et al. [17] and our findings in this retrospective analysis would both suggest a clinical benefit of anticoagulation on liver dysfunction in cirrhosis, these effects have to be confirmed in larger prospective studies in patients with cirrhosis with and without PVT. The monitoring of traditional anticoagulation in patients with cirrhosis represents a clinical challenge [24, 25] and while DOACs seem 
to be attractive alternative options, their efficacy and safety in cirrhosis has yet to be evaluated [26].

In conclusion, our findings support the use of AC in patients with non-malignant PVT, since AC is not only safe and associated with superior PVT regression/ resolution rates, but might also decrease hepatic inflammation and improve liver synthesis.

Acknowledgements We want to thank Prof. Dr. Ingrid Pabinger, head of the homeostaseology outpatient department of the Medical University of Vienna for her helpful comments on this manuscript.

Funding Open access funding provided by Medical University of Vienna.

\section{Compliance with ethical guidelines}

Conflict of interest B. Scheiner received travel support from Gilead. T. Bucsics received travel support from Gilead, BMS, Roche, Bayer and AbbVie. P. Schwabl received travel support from AbbVie, Gilead, Roche, Bayer and honoraria for lectures from Boehringer Ingelheim, Roche and MSD. P. R. Stammet, S. Pokorny, A. Brichta, J. Thaler, and K. Lampichler have nothing to disclose. A. Ba-Ssalamah received honoraria for lectures and consultancy from Bayer and Siemens. C. Ay received honoraria for lectures and advisory boards from Bayer, Boehringer/Ingelheim, Daiichi Sankyo and BMS/ Pfizer. A. Ferlitsch has served as a speaker and/or consultant and/or advisory board member for AbbVie, Gilead and Intercept and owns a patent on a catheter for the measurement of hepatic venous pressure gradient. M. Trauner received grants from MSD, honoraria for consulting from AbbVie, Gilead, Janssen and MSD, payments for lectures from Gilead, MSD, and Roche, as well as travel support from Gilead. M. Mandorfer received honoraria for consulting from Janssen, payments for lectures from Bristol-Myers Squibb, Janssen, and Roche, as well as travel support from AbbVie, Gilead, MSD, and Roche. T. Reiberger received payments for lectures from Roche, MSD, W. L. Gore, and Boehringer-Ingelheim, consulting fees from Abbvie, travel support from Gilead, MSD, and Roche as well as unrestricted research grants from Abbvie, Philips, Phenex Pharmaceuticals, W. L. Gore and BoehringerIngelheim.

Ethical standards This retrospective analysis was conducted according to the ethical principles of the Declaration of Helsinki and was approved by the ethics committee of the Medical University of Vienna (EKNR.1739/2016).

Open Access This article is distributed under the terms of the Creative Commons Attribution 4.0 International License (http://creativecommons.org/licenses/by/4.0/), which permits unrestricted use, distribution, and reproduction in any medium, provided you give appropriate credit to the original author(s) and the source, provide a link to the Creative Commons license, and indicate if changes were made.

\section{References}

1. Blachier M, Leleu H, Peck-Radosavljevic M, Valla DC, Roudot-Thoraval F. The burden of liver disease in Europe: a review of available epidemiological data. J Hepatol. 2013;58(3):593-608.

2. Ge PS, Runyon BA. Treatment of patients with cirrhosis. NEngl J Med. 2016;375(8):767-77.
3. de Franchis R, Baveno VIF. Expanding consensus in portal hypertension: report of the Baveno VI Consensus Workshop: stratifying risk and individualizing care for portal hypertension. J Hepatol. 2015;63(3):743-52.

4. Sogaard KK, Horvath-Puho E, Gronbaek H, Jepsen P, Vilstrup H, Sorensen HT. Risk of venous thromboembolism in patients with liver disease: a nationwide population-based case-control study. Am J Gastroenterol. 2009;104(1):96-101.

5. Tripodi A, Mannucci PM. The coagulopathy of chronic liver disease. NEngl J Med. 2011;365(2):147-56.

6. Rodriguez-Castro KI, Porte RJ, Nadal E, Germani G, Burra P, Senzolo M. Management of nonneoplastic portal vein thrombosis in the setting of liver transplantation: a systematic review. Transplantation. 2012;94(11):1145-53.

7. Ogren M, Bergqvist D, Bjorck M, Acosta S, Eriksson H, Sternby NH. Portal vein thrombosis: prevalence, patient characteristics and lifetime risk: a population study based on 23,796 consecutive autopsies. World J Gastroenterol. 2006;12(13):2115-9.

8. Luca A, Caruso S, Milazzo M, Marrone G, Mamone G, Crino F, et al. Natural course of extrahepatic nonmalignant partial portalvein thrombosis in patients with cirrhosis. Radiology. 2012;265(1):124-32.

9. Nery F, Chevret S, Condat B, de Raucourt E, Boudaoud $\mathrm{L}$, Rautou PE, et al. Causes and consequences of portal vein thrombosis in 1,243 patients with cirrhosis: results of a longitudinal study. Hepatology. 2015;61(2):660-7.

10. Senzolo M, MSartori T, Rossetto V, Burra P, Cillo U, Boccagni $\mathrm{P}$, et al. Prospective evaluation of anticoagulation and transjugular intrahepatic portosystemic shunt for the management of portal vein thrombosis in cirrhosis. Liver Int. 2012;32(6):919-27.

11. Francoz C, Belghiti J, Vilgrain V, Sommacale D, Paradis V, Condat B, et al. Splanchnic vein thrombosis in candidates for liver transplantation: usefulness of screening and anticoagulation. Gut. 2005;54(5):691-7.

12. Amitrano L, Guardascione MA, Menchise A, Martino R, Scaglione M, Giovine S, et al. Safety and efficacy of anticoagulation therapy withlow molecularweightheparin for portal vein thrombosis in patients with liver cirrhosis. JClin Gastroenterol. 2010;44(6):448-51.

13. Delgado MG, SeijoS, Yepes I, AchecarL, Catalina MV, GarciaCriado A, et al. Efficacy and safety of anticoagulation on patients with cirrhosis and portal vein thrombosis. Clin Gastroenterol Hepatol. 2012;10(7):776-83.

14. Werner KT, Sando S, Carey EJ, Vargas HE, Byrne TJ, Douglas $\mathrm{DD}$, et al. Portal vein thrombosis in patients with end stage liver disease awaiting liver transplantation: outcome of anticoagulation. Dig Dis Sci. 2013;58(6):1776-80.

15. European Association for the Study of the Liver. EASL clinical practice guidelines: vascular diseases of the liver. J Hepatol. 2016;64(1):179-202. Electronic address: easloffice@easloffice.eu.

16. Cerini F, Vilaseca M, Lafoz E, Garcia-Irigoyen O, GarciaCaldero H, Tripathi DM, et al. Enoxaparin reduces hepatic vascular resistance and portal pressure in cirrhotic rats. JHepatol. 2016;64(4):834-42.

17. Villa E, Camma C, Marietta M, Luongo M, Critelli R, Colopi $\mathrm{S}$, et al. Enoxaparin prevents portal vein thrombosis and liver decompensation in patients with advanced cirrhosis. Gastroenterology. 2012;143(5):1253-1260.e1-4.

18. de Franchis R, Baveno VF. Revising consensus in portal hypertension: report of the Baveno $\mathrm{V}$ consensus workshop on methodology of diagnosis and therapy in portal hypertension. JHepatol. 2010;53(4):762-8. 
19. Cui SB, Shu RH, Yan SP, Wu H, Chen Y, Wang L, et al. Efficacy and safety of anticoagulation therapy with different doses of enoxaparin for portal vein thrombosis in cirrhotic patients with hepatitis B. Eur J Gastroenterol Hepatol. 2015;27(8):914-9.

20. D'Amico G, Garcia-Tsao G, Pagliaro L. Natural history and prognostic indicators of survival in cirrhosis: a systematic review of 118 studies. JHepatol. 2006;44(1):217-31.

21. Zipprich A, Garcia-Tsao G, Rogowski S, Fleig WE, Seufferlein T, Dollinger MM. Prognostic indicators of survival in patients with compensated and decompensated cirrhosis. Liver Int. 2012;32(9):1407-14.

22. D'Amico G, Morabito A, Pagliaro L, Marubini E. Survival and prognosticindicatorsin compensated and decompensated cirrhosis. Dig Dis Sci. 1986;31(5):468-75.

23. Intagliata NM, Henry ZH, Maitland H, Shah NL, Argo CK, Northup PG, et al. Direct oral anticoagulants in cirrhosis patients pose similar risks of bleeding when compared to traditional anticoagulation. Dig Dis Sci. 2016;61(6):1721-7.

24. TripodiA, PrimignaniM, BrahamS, ChantarangkulV,Clerici M, Moia M, et al. Coagulation parameters in patients with cirrhosis and portal vein thrombosis treated sequentially with low molecular weight heparin and vitamin K antagonists. Dig Liver Dis. 2016;48(10):1208-13.

25. Tripodi A, Primignani M, Lemma L, Chantarangkul V, Dell'Era A, Iannuzzi F, et al. Detection of the imbalance of procoagulant versus anticoagulant factors in cirrhosis by a simple laboratory method. Hepatology. 2010;52(1):249-55.

26. Intagliata NM, Maitland H, Caldwell SH. Direct oral anticoagulants in cirrhosis. Curr Treat Options Gastroenterol. 2016;14(2):247-56. 